\title{
Bones, blood and steel: How bioelectrical impedance analysis is affected by hip fracture and surgical implants
}

\author{
Ole Martin Steihaug ${ }^{1,5}$, Bård Bogen ${ }^{1,2}$, Målfrid Holen Kristoffersen ${ }^{3}$ and Anette Hylen Ranhoff ${ }^{1,4}$ \\ 1. Haraldsplass Deaconess Hospital, Bergen, Norway \\ 2. Bergen University College, Bergen, Norway \\ 3. Department of Orthopedics, Haukeland University Hospital, Bergen, Norway \\ 4. Institute of Clinical Science, University of Bergen, Bergen, Norway \\ 5. E-mail any correspondence to: osteihaug@gmail.com (OMS)
}

\begin{abstract}
Introduction: Bioelectrical impedance analysis (BIA) is in widespread use, but there is uncertainty about its validity in patients with metal implants or after acute hip fracture and surgery. We aimed to investigate the use of single frequency tetrapolar BIA in patients with hip fracture by answering the following questions: 1 ) Are BIA measurements affected by recent hip fracture and surgical repair? 2) Are BIA measurements affected by the presence of metal implants used in hip fracture surgery?

Setting: Two hospitals in Bergen, Norway.

Participants: A convenience sample of 203 acute hip fracture patients.

Methods: Participants had their body composition measured by single frequency, tetrapolar BIA on the fractured and unfractured side of the body in the immediate postoperative period and at follow-up three months after hip fracture. Measurements from fractured and unfractured side and measurements in hospital and at follow-up were compared. BIA readings for hips treated with cannulated screws, compression hip screw and hip arthroplasty were compared.
\end{abstract}

Results: Resistance was lower on the side of the fractured hip compared to the unfractured side postoperatively, but not at follow-up. BIA readings did not differ by type of surgical implant. Conclusion: Recent fracture and surgery influences single frequency tetrapolar BIA resistance. The presence of surgical implants in the hip do not affect BIA measurements. If BIA is used in acute hip fracture patients, the contralateral side to the fracture should be measured.
Keywords: Hip fracture, surgical implants, bioelectrical impedance analysis

\section{Introduction}

Bioelectrical impedance analysis (BIA) is a popular tool for determining body composition (1). BIA is painless, requires minimal mobility by the person being measured, is portable and does not expose users to radiation. It is found in a range of inexpensive consumer-targeted devices such as bathroom scales, it is used by healthcare workers to determine the health and nutritional status of persons (2) and it is in use in large population based studies of body composition (3). BIA has been validated in comparison with other methods of determining body composition such as Dual Energy x-ray absorptiometry (DXA), underwater weighing and Magnetic Resonance Imaging $(4,5)$. BIA has been validated prospectively as a method to determine elevated risk associated with adverse body composition profiles (6). We have previously used data from this study to validate BIA for determining muscle mass in hip fracture patients (7). Patients with acute hip fracture often have difficulty in walking and getting on to the examination table of a DXA, CT or MRI machine. BIA could be a valuable tool to investigate body composition in acute hip fracture patients. There are still concerns that BIA is not sufficiently validated since BIA is 
influenced by health status and should be validated separately in each population (8-11).

Many older persons live with surgical implants. It has been estimated that in the population aged $50+$ in the UK there is a lifetime risk of 7-12\% for receiving a total hip arthroplasty and 8-11\% for total knee replacement (12). The validity of BIA to determine body composition in individuals with surgical implants is not determined, but is widely considered to be problematical $(13,14)$.

When the proximal femur is fractured, the tension of surrounding tendons and muscle will often lead to dislocation of the fracture ends, which prevents skeletal healing. Surgical treatment of hip fracture involves surgical implants to fix the ends of the femur in their anatomical correct position to enable healing. There is a multitude of different surgical implants used for hip fracture repair, but the implants most commonly used can be categorized in three broad categories: hemiarthroplasty and total hip arthroplasty, cannulated screws and hip compression screws (Fig. 1 and 2).

Fracture and surgery are associated with discontinuities of the tissue spaces and edema. The surgical implants are mainly made of metals such as steel, titanium, cobalt and chromium with use of other materials such as ceramics, hydroxyapatites and polyethylenes. These changes can potentially increase or decrease the electrical conductance, and it is difficult to predict how they will affect BIA measurements. If BIA is to be useful in the large group of acute hip fracture patients, or other patient groups undergoing surgery, it is important to determine the influence of fracture, surgery and surgical implants on BIA readings. The aim of this study is to answer the following research questions: 1) Are BIA measurements affected by recent fracture and surgical repair? 2) Are BIA measurements affected by the presence of metal implants used in hip fracture surgery?

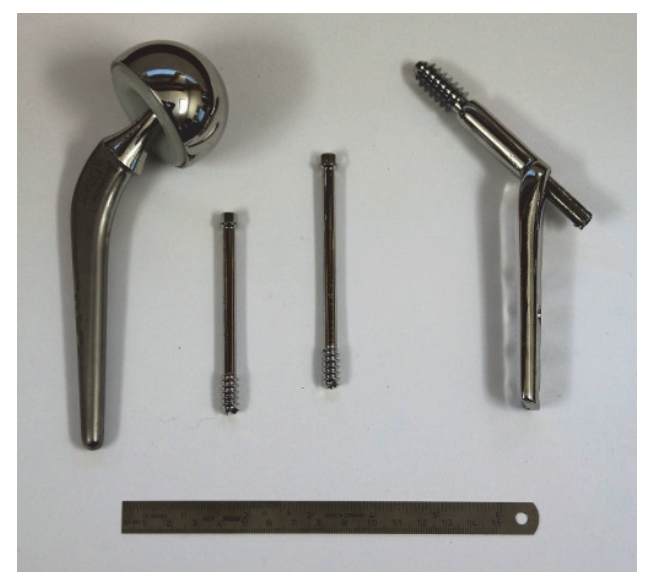

Photo: Ole Martin Steihaug.

Figure 1: Surgical implants used for hip fracture repair. From left: hemiarthroplasty of the hip, two cannulated screws and a compression hip screw.
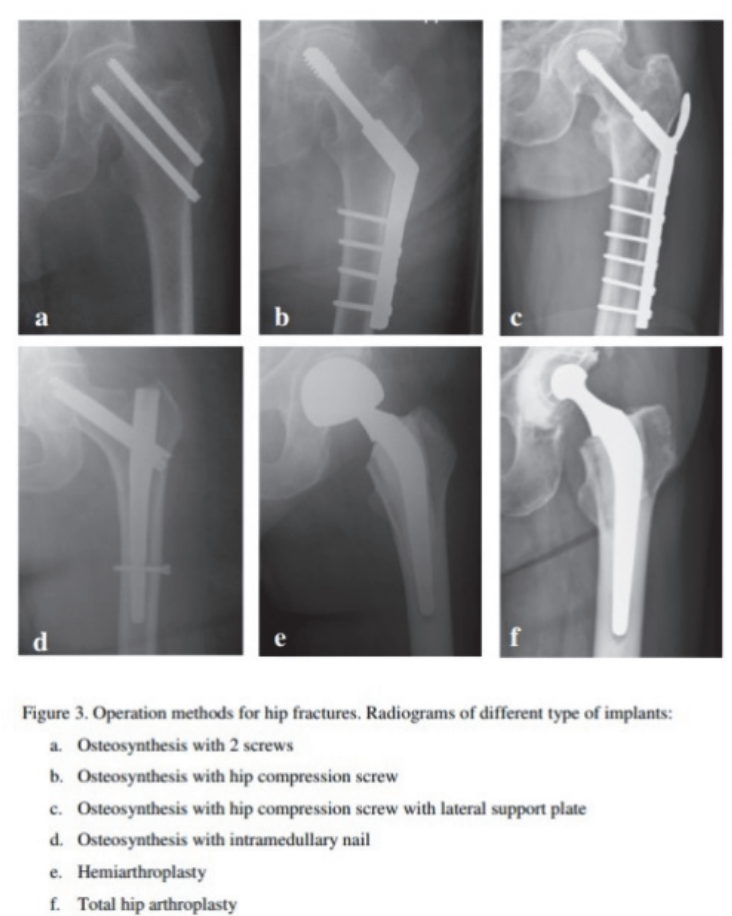

Fig. 2: Plain film X-rays of hip fractures treated with different surgical implants. From Gjertsen (15). Reproduced with permission.

\section{Methods}

Patients admitted to hospital with suspected hip fracture were screened for inclusion in the study when the research staff was present on the hospital wards. Participants had to undergo surgical repair of acute hip fracture and be aged $\geq 65$ years, be ambulatory before the fracture, give informed consent, have an estimated remaining life expectancy of $>3$ months and not have any disease of bone apart from osteoporosis or osteomalacia. Participants could not suffer from delirium, severe pain, have acute respiratory failure or be in shock at inclusion, but could develop these after inclusion. Participants were excluded if they had pacemakers or implanted defibrillators since these could be affected by BIA measurements. Patients could have preexisting surgical implants. Patients who were permanent residents of skilled nursing homes were not eligible for inclusion.

\section{Anthropometry}

Patients were weighed with indoor clothing. Height was primarily determined by wall mounted stadiometer. Some of the patients measured in hospital were not able to have their standing height measured. In these cases, self-reported height or length in bed was measured. Length in bed was measured while supine in a hospital bed, measured from heel to crown. Weight was determined by the available scale, often a chair-weight while in hospital.

\section{Bioelectrical impedance analysis}

BIA measurements in hospital were performed after hip fracture surgery when the patients had returned to the orthopedic ward from the recovery ward. The BIA resistance 
and reactance (ohms) was obtained using single frequency tetrapolar BIA (RJL quantum systems III, RJL systems, USA) with an operating frequency of $50 \mathrm{kHz}$ at $425 \mu \mathrm{A}$, and at 400 $\mu \mathrm{A}$ and $50 \mathrm{kHz}$ (Body impedance analyzer BIA $101 \mathrm{ASE}$, Akern Srl, Italy). Electrodes (RJL systems, USA) were placed on the skin at the wrist and ankle with participant supine in a hospital bed. The Quantum systems III was calibrated before measurements. Arms and legs were slightly spread so that they were not in contact with other parts of the body. The skin was not cleaned before applying the electrodes unless it was visibly or palpably dirty. Rings, bracelets and wristwatches were removed, if possible. Patients were not fasting and there was no systematic bladder voiding. Patients were measured first on one half of the body, then immediately afterwards on the other half using a new set of electrodes and by the same operator and using the same BIA device. All BIA measurements were performed by research nurses or the study physicians (MHK, OMS). We limited the sample to participants who had BIA readings from both fractured and unfractured side and did not have surgical implants in the opposite hip. All measurements were performed indoors with stable humidity and temperature between readings. The decision to measure both sides of the body was by protocol amendment after inclusion had started.

\section{Follow-up}

Participants were invited for a follow-up examination three months after admission to hospital. On the same day patients were measured by whole body DXA, BIA and anthropometry. Scheduling of the follow-up appointment was flexible to increase attendance and minimize the inconvenience for participants.

\section{Statistics}

The resistance of the fractured and unfractured hip was analyzed by mean difference with limits of agreement according to the method of Bland and Altman (16). The Bland-Altman methods was chosen because we considered the measurements of the fractured and the unfractured side of the body two alternative methods for determining wholebody muscle mass. We assumed that BIA measurements on the side of the unfractured hip were not affected by the surgery or surgical implants of the fractured hip. We also assumed that any effect of fracture or surgery would be reduced at follow-up compared to while in hospital. Participants with pre-existing surgical implants in the unfractured hip were excluded from analysis. Resistance and reactance of the fractured side was compared with the unfractured side using paired T-test, in hospital and at follow-up, for a total of four comparisons. For determining the influence of surgical implants on resistance and reactance, the difference between the resistance and reactance of the fractured and unfractured hip was analyzed by analysis of variance using category of surgical implant. The categories were hip screws, compression hip screw and hip arthroplasty. The arthroplasty category constituted both hip hemiarthroplasty and total hip arthroplasty. $\mathrm{P} \leq 0.05$ was considered statistically significant. Analysis was by Stata Statistical Software: Release 14. StataCorp LP, USA.

\section{Ethics}

The research was conducted according to the declaration of Helsinki. Participants were given written and verbal information about the study on the first day and were asked to sign the consent form on a subsequent day. This enabled time for deliberation and to consult their next of kin.

Participants were included when they had been mobilized to sitting upright and with adequate pain relief. In situations where the capacity for consent was in doubt, experienced medical doctors and the rest of the team treating the patients were consulted.

\section{Results}

Table 1: Characteristics of the participants.

\begin{tabular}{lrr}
\hline Age, years (SD) & 80 & $(8)$ \\
Female, $\mathrm{n}(\%)$ & 151 & $(76)$ \\
Right sided fracture, $\mathrm{n}(\%)$ & 109 & $(54)$ \\
\hline & & \\
Type of surgical implant, $\mathbf{n}(\%)$ & & \\
\hline Cannulated screws & 44 & $(22)$ \\
Hemiarthroplasty & 62 & $(31)$ \\
Hip compression screw & 83 & $(41)$ \\
Total hip arthroplasty & 8 & $(4)$ \\
Femoral nail & 6 & $(3)$ \\
\hline
\end{tabular}

\begin{tabular}{|c|c|c|c|c|c|c|}
\hline \multirow[b]{2}{*}{ Height (SD), cm } & \multicolumn{2}{|c|}{ In hospital } & \multicolumn{4}{|c|}{ At follow-up } \\
\hline & 166 & (10) & $n=136$ & 164 & (9) & $n=162$ \\
\hline Weight (SD), kg & 66 & (16) & $n=136$ & 64 & (14) & $n=162$ \\
\hline \multicolumn{7}{|l|}{ Fractured hip } \\
\hline Resistance (SD), Ohm & 491 & (95) & $n=134$ & 555 & (95) & $n=157$ \\
\hline Reactance (SD), Ohm & 46 & (31) & $n=134$ & 49 & (13) & $n=157$ \\
\hline \multicolumn{7}{|l|}{ Unfractured hip } \\
\hline Resistance (SD), Ohm & 527 & (99) & $n=131$ & 552 & (89) & $n=158$ \\
\hline Reactance (SD), Ohm & 53 & (51) & $n=131$ & 53 & $(23)$ & $n=158$ \\
\hline
\end{tabular}

Table 2: Pairwise comparisons of BIA readings on fractured and unfractured hips.

\begin{tabular}{lrrrrrr}
\hline & Fractured & \multicolumn{3}{c}{ Unfractured } & P-value & N \\
\hline $\begin{array}{l}\text { Resistance } \\
\text { Hospital }\end{array}$ & 496 & $(98)$ & 527 & $(101)$ & 0.0007 & 81 \\
Follow-up & 553 & $(98)$ & 550 & $(92)$ & 0.4 & 134 \\
\hline Reactance & & & & & & \\
Hospital & 45 & $(20)$ & 56 & $(64)$ & 0.068 & 81 \\
Follow-up & 49 & $(14)$ & 52 & $(23)$ & 0.061 & 134 \\
\hline
\end{tabular}

During the period of inclusion 843 patients were operated on for hip fracture, 203 were included and 163 returned for follow-up. All participants were Caucasian. BIA was performed at a median of 5 (IQR 4-7) days after hip fracture, which was a median 4 (IQR 2-5) days after surgery. 
The number of days from admission to follow-up was a median 112 days (IQR 98-133).

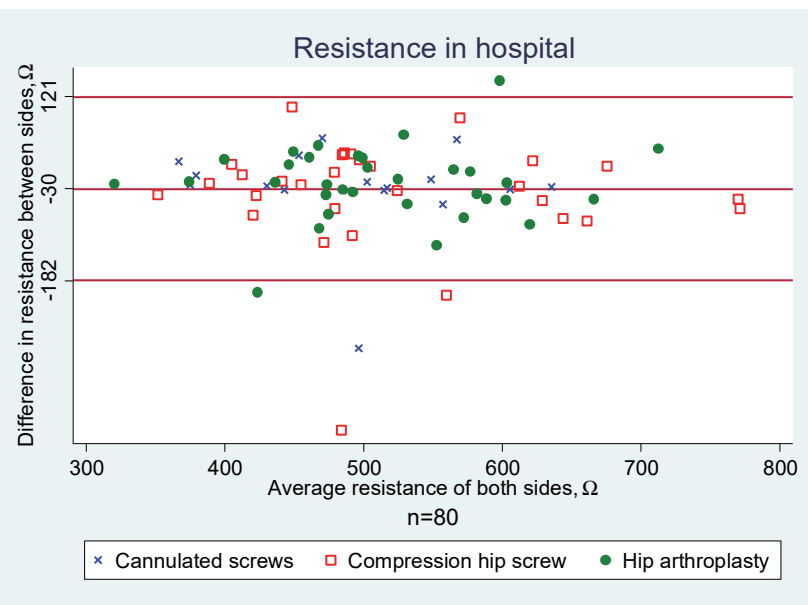

Fig. 3: Bland-Altman plot of difference in resistance between fractured and unfractured hip postoperatively after hip fracture. Red lines are the mean, upper and lower limits of agreement (mean bias \pm 1.96 standard deviations).

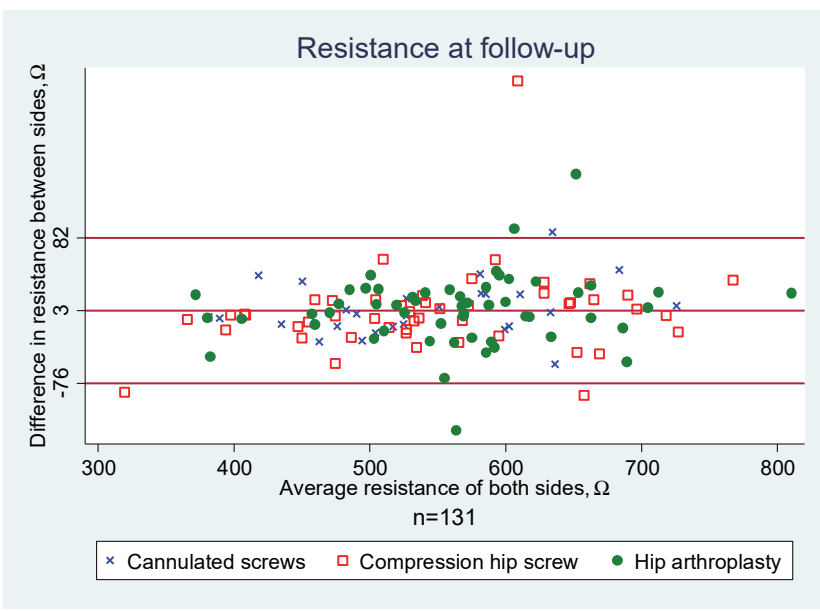

Fig. 4: Bland-Altman plot of difference in resistance between fractured and unfractured hip 3 months after hip fracture. Red lines are the mean, upper and lower limits of agreement (mean bias \pm 1.96 standard deviations).

Change from hospital to follow-up \& Sorted by difference in resistance between hips in hospital

\&

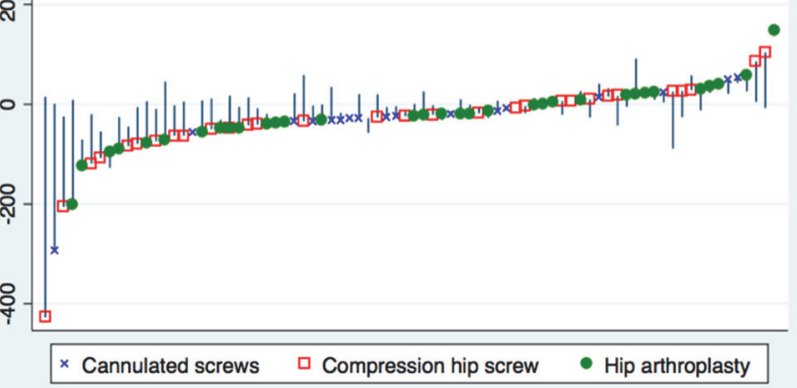

Fig. 5: Change in difference between fractured and unfractured hip from hospital to follow-up. Symbols indicates type of surgical implant and difference between fractured and unfractured hip in hospital. The vertical line indicates the magnitude of the change in difference between fractured and unfractured hip from hospital to follow-up.
In hospital, resistance on the side of the body with the fractured hip was lower than the side with the unfractured hip, 496 (SD 98) ohm vs 527 (SD 101) ohm, P=0.0007. Table 2. The limit of agreement was wider in hospital compared to at follow-up, indicating that fracture and surgery affected BIA measurements. Figure 3 and 4 . The difference in resistance or reactance between fractured and nonfractured hip did not vary by type of surgical implant, either during hospital stay or at follow-up.

\section{Discussion}

Our results show that BIA is not affected by the type of surgical implants of the hip. There is a statistically and clinically relevant effect of fracture and/or surgery on resistance in the fractured hip, including important outliers. The clinical relevance is illustrated by the mean difference and limit of agreement of appendicular lean mass determined by BIA on the fractured and the unfractured side, using the equation of Sergi et al (17). In hospital the mean difference is $0.2 \mathrm{~kg}(\mathrm{n}=57)$, with a large limit of agreement of -5.7 to $6.0 \mathrm{~kg}$. At follow-up the limit of agreement is narrower, -3.0 to $2.6 \mathrm{~kg}$, with a mean bias of $-0.2 \mathrm{~kg}$. A clinically relevant change in appendicular lean mass is of a magnitude of $1 \mathrm{~kg}$ (7). A practical solution to this problem is to measure the unfractured side.

We performed the BIA measurements in a correct and competent manner, but we did not adhere to all recommendations for increasing precision of the BIA measurements. Some of these recommendations are controversial and the strict adherence to all such recommendations is impractical (18).

We found that resistance is affected by recent fracture or surgery. Our results do not inform us if it is the fracture, the surgery or a combination that influences BIA readings. A future study which examined BIA readings after fracture, but before surgery could possibly answer that question. We are not aware of previous studies on the effect of fracture and surgery on BIA measurements. A study by Villani et al used BIA in patients with acute hip fracture (19), but they performed all measurements on the right side, irrespective of the side of fracture. A study by Gonzalez-Montalvo et al used BIA after hip fracture and before surgery, but they only measured BIA on the contralateral side to the fracture (20).

Our results indicate that BIA is not affected by type of surgical implant of the three categories we examined. Hip arthroplasty, cannulated screws and compression hip screws have different masses and placement in the hip region. We believe that if the shape and the approximate shape of the different tissues of bone, fat and skeletal muscle mass are unchanged the type of surgical implant does not matter. It seems likely that more severe traumatic injuries where the shape of the hip is fundamentally changed or a tissue compartment is removed can result in larger changes in resistance or reactance. 
It is possible that other acute changes could influence BIA readings in hospital. Patients could suffer from fluid and electrolyte disturbances due to illness, dehydration or fluid retention due to heart failure. We do not have information about these factors.

Even if the included population is not generalizable to all hip fracture patients, we believe the edema and tissue destruction associated with fracture and surgery is representative of all hip fracture patients. The surgical implants used in this study, predominantly compression hip screws, hip arthroplasty and cannulated screws, are the same as most patients with hip fracture are surgically treated.

This is the first time BIA is critically examined in a setting of acute tissue destruction and our findings indicate that care must be taken if BIA is to be used in similar settings, such as in other forms of surgery or trauma.

We note that the wide dispersion of results with outliers continue to be a problem for precise BIA measurements at an individual level.

\section{Conclusion}

Tetrapolar single frequency BIA is affected by recent surgery and fracture, but not by type of surgical implant. BIA can be used to determine body composition in patients who have suffered hip fracture. We recommend measuring the contralateral side to the hip fracture in the immediate postoperative period. This supports using BIA to determine body composition in patients with surgical implants.

\section{Acknowledgements}

We are grateful for the time and effort donated by the participants. The original research idea was by Professor Lars B. Engesæter. Britt Pedersen and Cathrine Sande were research nurses on the project. Funding was by Haraldsplass Deaconess Hospital, Bergen, Norway and The Western Norway Regional Health Authority, Stavanger, Norway.

\section{Conflicts of interest}

The authors declare no conflict of interest.

\section{References}

1. Haverkort EB, Reijven PL, Binnekade JM, de van der Schueren MA, Earthman CP, Gouma DJ, et al. Bioelectrical impedance analysis to estimate body composition in surgical and oncological patients: a systematic review. European journal of clinical nutrition. 2015;69(1):3-13.

https://doi.org/10.1038/ejcn.2014.203

2. Bruyere $O$, Beaudart C, Reginster JY, Buckinx F, Schoene D, Hirani $V$, et al. Assessment of muscle mass, muscle strength and physical performance in clinical practice: An international survey. Eur Geriatr Med. 2016;7(3):243-6. https://doi.org/10.1016/j.eurger.2015.12.009

3. Bohm A, Heitmann BL. The use of bioelectrical impedance analysis for body composition in epidemiological studies.
European journal of clinical nutrition. 2013;67(S1):S79-S85. https://doi.org/10.1038/ejcn.2012.168

4. Mijnarends DM, Meijers JM, Halfens RJ, ter Borg S, Luiking YC, Verlaan $\mathrm{S}$, et al. Validity and reliability of tools to measure muscle mass, strength, and physical performance in community-dwelling older people: a systematic review. J Am Med Dir Assoc. 2013;14(3):170-8. https://doi.org/10.1016/j.jamda.2012.10.009

5. Janssen I, Heymsfield SB, Baumgartner RN, Ross R. Estimation of skeletal muscle mass by bioelectrical impedance analysis. Journal of applied physiology. 2000;89(2):465-71.

6. Srikanthan $P$, Karlamangla AS. Muscle mass index as a predictor of longevity in older adults. Am J Med. 2014;127(6):547-53. https://doi.org/10.1016/j.amjmed.2014.02.007

7. Steihaug OM, Gjesdal CG, Bogen B, Ranhoff AH. Identifying Low Muscle Mass in Patients with Hip Fracture: Validation of Biolectrical Impedance Analysis and Anthropometry Compared to Dual Energy X-ray Absorptiometry. J Nutr Health Aging. 2016;20(7):685-90. https://doi.org/10.1007/s12603-016-0686-1

8. Vellas B, Fielding R, Miller R, Rolland Y, Bhasin S, Magaziner J, et al. Designing Drug Trials for Sarcopenia in Older Adults with Hip Fracture - a Task Force from the International Conference Onfrailty and Sarcopenia Research (Icfsr). J Frailty Aging. 2014;3(4):199-204.

9. Cruz-Jentoft AJ, Baeyens JP, Bauer JM, Boirie Y, Cederholm T, Landi F, et al. Sarcopenia: European consensus on definition and diagnosis: Report of the European Working Group on Sarcopenia in Older People. Age Ageing. 2010;39(4):412-23. https://doi.org/10.1093/ageing/afq034

10. Tosato M, Marzetti E, Cesari M, Savera G, Miller RR, Bernabei R, et al. Measurement of muscle mass in sarcopenia: from imaging to biochemical markers. Aging Clin Exp Res. 2017. https://doi.org/10.1007/s40520-016-0717-0

11. Kyle UG, Bosaeus I, De Lorenzo AD, Deurenberg P, Elia M, Gomez JM, et al. Bioelectrical impedance analysis--part I: review of principles and methods. Clinical nutrition. 2004;23(5):1226-43. https://doi.org/10.1016/j.clnu.2004.06.004

12. Culliford DJ, Maskell J, Kiran A, Judge A, Javaid MK, Cooper C, et al. The lifetime risk of total hip and knee arthroplasty: results from the UK general practice research database. Osteoarthritis and cartilage. 2012;20(6):519-24. https://doi.org/10.1016/j.joca.2012.02.636

13. Gonzalez-Correa C, Caicedo-Eraso J, editors. Bioelectrical impedance analysis (BIA): a proposal for standardization of the classical method in adults. Journal of Physics: Conference Series; 2012: IOP Publishing.

14. Kyle UG, Bosaeus I, De Lorenzo AD, Deurenberg P, Elia M, Manuel Gomez J, et al. Bioelectrical impedance analysis-part II: utilization in clinical practice. Clinical nutrition. 2004;23(6):1430-53. https://doi.org/10.1016/j.clnu.2004.09.012

15. Gjertsen J-E. Surgical treatment of hip fractures in Norway. The Norwegian Hip Fracture Register. 2009. 
16. Bland JM, Altman DG. Measuring agreement in method comparison studies. Stat Methods Med Res. 1999;8(2):135-60. https://doi.org/10.1191/096228099673819272

17. Sergi G, De Rui M, Veronese N, Bolzetta F, Berton L, Carraro S, et al. Assessing appendicular skeletal muscle mass with bioelectrical impedance analysis in free-living Caucasian older adults. Clinical nutrition. 2014.

18. Androutsos O, Gerasimidis K, Karanikolou A, Reilly JJ, Edwards CA. Impact of eating and drinking on body composition measurements by bioelectrical impedance. Journal of human nutrition and dietetics : the official journal of the British Dietetic Association. 2015;28(2):165-71.

https://doi.org/10.1111/jhn.12259
19. Villani AM, Miller M, Cameron ID, Kurrle S, Whitehead C, Crotty M. Body composition in older community-dwelling adults with hip fracture: portable field methods validated by dual-energy $X$ ray absorptiometry. Br J Nutr. 2012:1-11.

20. Gonzalez-Montalvo II, Alarcon T, Gotor P, Queipo R, Velasco R, Hoyos R, et al. Prevalence of sarcopenia in acute hip fracture patients and its influence on short-term clinical outcome. Geriatrics \& gerontology international. 2016;16(9):1021-7. https://doi.org/10.1111/ggi.12590 